\title{
Carcinoma of the esophagus
}

\author{
Prognostic significance of histologic type
}

Previous investigators have reported that in patients with esophageal carcinoma tumor cell type affects prognosis. A retrospective analysis of 258 patients, from 1985 to 1991, undergoing curative esophagogastrectomy for adenocarcinoma $(n=134)$ or squamous cell carcinoma $(n=124)$ was performed to test the hypothesis that histologic cell type is an independent prognostic factor and to identify other predictors of survival after resection. The actuarial overall survival $(p=0.16)$ and disease-specific survival $(p=0.68$ ) were similar for adenocarcinoma (median overall survival $=27$ months) and squamous cell carcinoma (median overall survival $=22$ months). Univariate analysis identified $\mathbf{T}$ stage, $\mathbf{N}$ stage, number of diseased nodes, tumor differentiation, tumor site, and blood transfusions as significant $(p<0.05)$ variables in predicting overall survival. The presence of Barrett's esophagus was not predictive of survival. Multivariate analysis demonstrated that $T$ stage $(p=0.006)$, N stage $(p=0.01)$, and number of diseased lymph nodes $(p=0.03)$ were independent predictors of overall survival. This analysis demonstrated that histologic type is not an independent variable for overall survival in patients undergoing curative esophagogastrectomy for carcinoma of the esophagus and gastroesophageal junction. Outcome is most strongly influenced by extent of disease defined by $T$ and N stage. (J THORAC CaRdIOVASC Surg 1995;109:130-9)

Michael D. Lieberman, MD (by invitation), Craig D. Shriver, MD (by invitation), Steven Bleckner, BS (by invitation), Michael Burt, MD, PhD, and the Thoracic and Gastric and Mixed Tumor Services, New York, N.Y.

$\mathrm{O}$ ver the past decade an increase in the prevalence of adenocarcinoma of the gastroesophageal junction and distal esophagus has been reported., 2 At Memorial Sloan-Kettering Cancer Center the proportion of gastric resections that encompassed the gastroesophageal junction has increased from $31 \%$ in 1985 to $53 \%$ in 1992 . Several investigators have suggested that histologic cell type affects prognosis in patients with esophageal carcinoma. ${ }^{3-10}$ In previous studies, adenocarcinoma has had a more adverse effect on survival than squamous cell carcinoma, and poorly differentiated carcinoma has had

From the Department of Surgery, Memorial Sloan-Kettering Cancer Center, New York, N.Y.

Read at the Seventy-fourth Annual Meeting of The American Association for Thoracic Surgery, New York, N.Y., April 24-27, 1994.

Address for reprints: Michael Burt, MD, PhD, Memorial SloanKettering Cancer Center, 1275 York Ave., New York, NY 10021.

Copyright (C) 1995 by Mosby-Year Book, Inc.

$0022-5223 / 95 \$ 3.00+0 \quad \mathbf{1 2 / 6 / 5 9 9 6 0}$ a more adverse effect than well or moderately differentiated carcinoma. ${ }^{3-10}$ However, many of these studies were univariate analyses and included patients treated over a period of two decades. This retrospective analysis was performed to test the hypothesis that histologic type is an independent prognostic variable and to identify other predictors of survival after potentially curative esophagogastrectomy in patients with esophageal carcinoma.

\section{Methods}

A computerized tumor registry of patients with the diagnosis of adenocarcinoma or squamous cell carcinoma of the gastroesophageal junction or esophagus treated at Memorial Sloan-Kettering Cancer Center from 1985 to 1991 was reviewed to obtain the records of 258 patients undergoing curative resection. A retrospective chart review was performed to identify perioperative factors that may influence overall survival after tumor resection. Follow-up was completed through office charts and telephone interviews. Categorical and continuous variables that were analyzed included age, sex, dysphagia, tobacco smoking, alcohol use, weight loss, operative procedure, splenectomy, blood transfusion, anastomotic leak, $\mathrm{T}$ stage, $\mathrm{N}$ 
stage, number of diseased lymph nodes, tumor differentiation, Barrett's esophagus, margins, tumor site, and preoperative chemotherapy or radiation therapy.

Curative resection was defined as the surgical removal of all gross tumor in the absence of distant metastases and included the removal of locoregional lymph nodes. Gastrointestinal continuity was reestablished by formation of a gastric tube and reanastomosis to the esophagus in the superior part of the mediastinum or neck. Tumor site was anatomically defined: proximal-cricopharyngeus to aortic arch; middle-extending from aortic arch to inferior pulmonary vein; distal-caudad to inferior pulmonary vein. Gastroesophageal junction tumors were included in this analysis if greater than $50 \%$ of the tumor mass was located in the esophagus.

To assess the dependence of the rate of death caused by esophageal cancer on explanatory variables, we measured time from the date of operation to the date of death or last follow-up information. Survival was estimated for each variable by the product-limit method of Kaplan and Meier. ${ }^{12}$ The log-rank test was used to check for dependence of survival on each categorical variable analyzed. ${ }^{13}$ A univariate proportional hazards regression was estimated and a likelihood ratio test was used to analyze the dependence of survival on each continuous variable. Proportional hazards regression was used to incorporate all of the explanatory variables in the same model. ${ }^{14}$ Forward stepwise procedure and likelihood ratio tests were used to select the variables with the greatest prognostic value. The statistical analysis was performed with the use of the SPSS statistical package (SPSS Inc., Chicago, Ill.). Frequency data were analyzed by the $\chi^{2}$ method. The critical significance level of $5 \%$ was chosen.

\section{Results}

This study included 258 patients undergoing curative resection of adenocarcinoma $(n=134)$ or squamous cell carcinoma $(n=124)$ of the esophagus and gastroesophageal junction. The median age was similar for patients having adenocarcinoma (63 years) and those having squamous cell carcinoma (64 years). The female/male sex ratio was 1:6.4 for adenocarcinoma and 1:1.6 for squamous cell tumors $(p<0.001)$. The most common presenting symptom was dysphagia (69\%), particularly for patients with squamous cell carcinoma (squamous $=86 \%$ and adenocarcinoma $=52 \%, p<0.001$ ). Weight loss, defined as greater than $10 \%$ loss from premorbid body weight, was more common in patients with squamous cell tumors $(56 \%)$ than in those with adenocarcinomas $(27 \%),(p<0.001)$. Most patients had a history of tobacco smoking (squamous $=82 \%$ and adenocarcinoma $=72 \%, p=0.80$ ) and alcohol use (squamous $=77 \%$ and adenocarcinoma $=56 \%$, $p=0.08$ ). Diagnosis was established by esophagogastroscopy and biopsy. The tumor was located in the proximal esophagus in $6 \%$, middle esophagus in
Table I. Comparison of the prevalence of prognostic variables according to cell type

\begin{tabular}{lccc}
\hline & \multicolumn{3}{c}{ Prevalence (\%) } \\
\cline { 2 - 3 } \multicolumn{1}{c}{ Characteristic } & Adenocarcinoma & $\begin{array}{c}\text { Squamous cell } \\
\text { carcinoma }\end{array}$ & $\begin{array}{c}p \text { Value } \\
\left(\chi^{2}\right)\end{array}$ \\
\hline $\begin{array}{l}\text { Advanced T stage } \\
\quad(T 3)\end{array}$ & 78 & 65 & 0.03 \\
Node diseased & 69 & 48 & 0.002 \\
$\geq 4$ Nodes diseased & 50 & 35 & 0.08 \\
Poorly differentiated & 46 & 33 & 0.06 \\
Blood transfusion & 46 & 59 & 0.04 \\
\hline
\end{tabular}

$21 \%$, and distal esophagus or gastroesophageal junction in $73 \%$. All adenocarcinomas $(n=134)$ were located in the distal esophagus or gastroesophageal junction.

Treatment was predominantly surgery alone $(n=$ 221 , although a small number of patients received preoperative chemotherapy $(n=19)$, radiation therapy $(n=12)$, or chemoradiation $(n=6)$. All patients receiving preoperative chemotherapy or radiation therapy, or both, had squamous cell carcinoma. The primary operative procedures were transthoracic esophagogastrectomy (79\%), transabdominal esophagogastrectomy (9\%), transhiatal esophagectomy (7\%), and pharyngoesophagectomy $(5 \%)$. Splenectomy was performed in $25 \%$ of patients with adenocarcinoma $(n=33)$ and $10 \%$ of patients with squamous cell carcinomas $(n=12)$ $(p<0.01)$. Histologic tumor-free margins were less than $2 \mathrm{~cm}$ in $42 \%$ of patients with adenocarcinoma and $17 \%$ of patients with squamous cell carcinoma $(p<0.0001)$. In-hospital mortality was $5 \%$ and was attributed to sepsis (pneumonia, $n=5$; anastomotic leak, $n=4)$, hemorrhage $(n=2)$, cardiac embolism $(n=1)$, and pulmonary embolism $(n=1)$. The perioperative deaths occurred in three patients with adenocarcinoma and 10 patients with squamous cell carcinoma $(p=0.06)$. Twenty-six anastomotic dehiscences occurred (10\%) (adenocarcinoma, $n=14$; squamous cell, $n=12 ; p=$ not significant).

Table I provides a comparison of the prevalence of prognostic variables identified by univariate analysis according to tumor type. Advanced $\mathrm{T}$ stage, presence of lymph node metastasis, four diseased lymph nodes or more, and poorly differentiated tumors were significantly more prevalent in patients with adenocarcinoma than in those with squamous cell carcinoma. However, patients undergoing resection for squamous cell tumors had a greater require- 


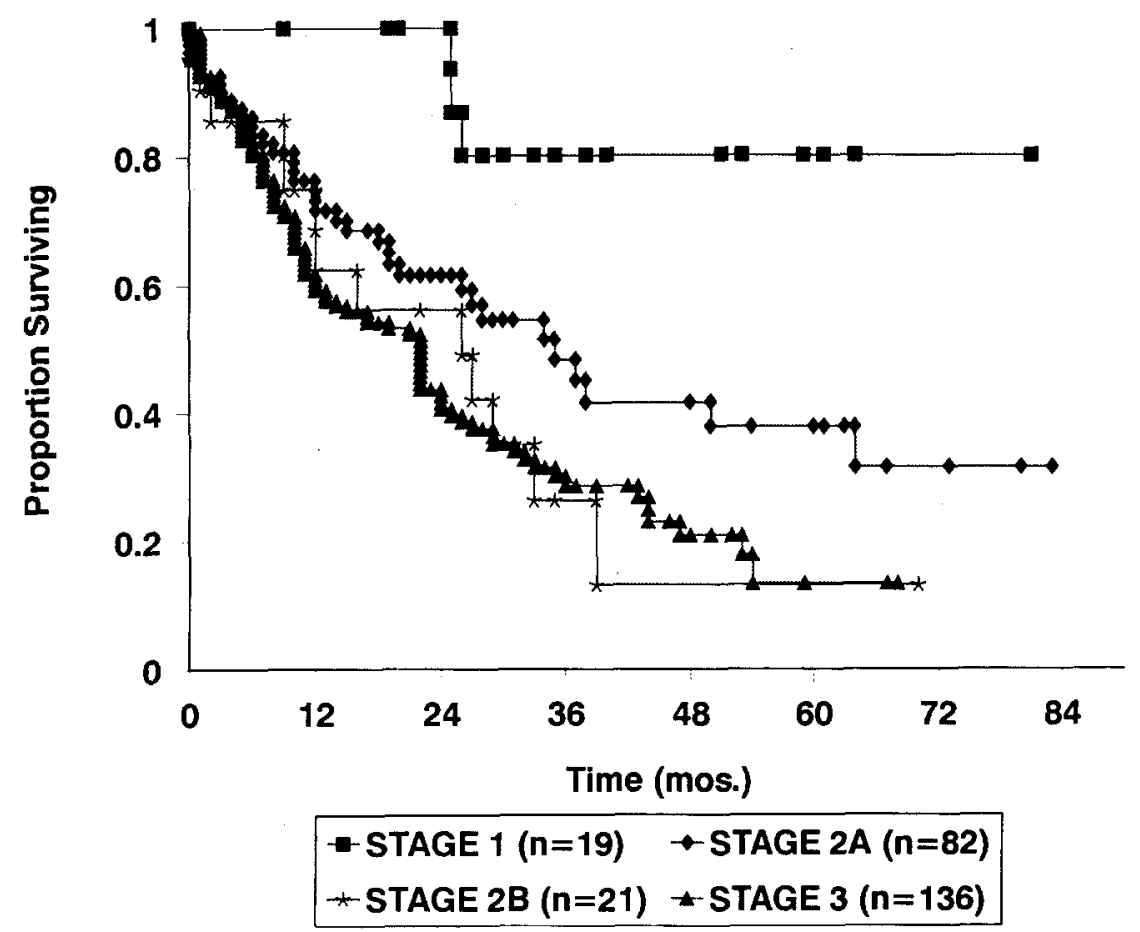

Fig. 1. Overall survival according to the staging system of the American Joint Committee on Cancer Staging ( 1 versus $2 \mathrm{~A}, p=0.006 ; 1$ versus $2 \mathrm{~B}, p=0.0006 ; 1$ versus $3, p=0.00007 ; 2 \mathrm{~A}$ versus $2 \mathrm{~B}, p=0.004$; $2 \mathrm{~A}$ versus $3, p=0.016$; and $2 \mathrm{~B}$ versus $3, p=0.89$ ).

Table II. Prognostic variables for overall survival after esophagogastrectomy for carcinoma of the esophagus or gastroesophageal junction

\begin{tabular}{|c|c|c|}
\hline Variable & $\begin{array}{c}\text { Three-year } \\
\text { survival } \\
(\%)\end{array}$ & $p$ Value* \\
\hline \multicolumn{3}{|l|}{ T stage } \\
\hline $\mathrm{T} 1$ & 64 & $\mathrm{~T} 1$ vs. $\mathrm{T} 2, p=0.18$ \\
\hline $\mathrm{T} 2$ & 56 & $\mathrm{~T} 2$ vs. T3, $p=0.03$ \\
\hline T3 & 30 & T1 vs. T3, $p=0.003$ \\
\hline \multicolumn{3}{|l|}{$N$ stage } \\
\hline No & 56 & N0 vs. N1, $p=0.00004$ \\
\hline $\mathrm{N} 1$ & 27 & \\
\hline \multicolumn{3}{|l|}{ No. of diseased nodes } \\
\hline 0 & 56 & 0 vs. $1-3, p=0.005$ \\
\hline $1-3$ & 37 & 1.3 vs. $\geq 4, p=0.04$ \\
\hline$\geq 4$ & 18 & 0 vs. $\geq 4, p<0.00001$ \\
\hline \multicolumn{3}{|l|}{ Tumor differentiation } \\
\hline $\begin{array}{l}\text { Well or moderately } \\
\text { differentiated }\end{array}$ & 41 & 0.05 \\
\hline Poorly differentiated & 34 & \\
\hline \multicolumn{3}{|l|}{ Site } \\
\hline Proximal & 45 & Proximal vs. middle, $p=0.71$ \\
\hline Middle & 29 & Middle vs. distal, $p=0.02$ \\
\hline Distal & 41 & Proximal vs. distal, $p=0.55$ \\
\hline \multicolumn{3}{|l|}{ Blood transfusion } \\
\hline No & 47 & 0.04 \\
\hline Yes & 31 & \\
\hline
\end{tabular}

"Univariate analysis according to log-rank test. ment for perioperative blood transfusion than did those with adenocarcinoma.

Survival. The actuarial overall survival for all patients $(n=258)$ was $68 \%, 39 \%$, and $27 \%$ at 1,3 , and 5 years, respectively. The overall median survival was 26 months with a median follow-up of 18 months. At the time of last follow-up 82 patients were alive with no evidence of disease, 36 patients were alive with disease, 127 patients had died of esophageal cancer, and 13 patients had died in the perioperative period. The 3-year overall survival according to the staging system of the American Joint Committee on Cancer Staging ${ }^{11}$ included stage $1,80 \%(n=19)$; stage $2 \mathrm{~A}, 48 \%(n=82)$; stage $2 \mathrm{~B}$, $26 \%(n=21)$; and stage 3, 29\% $(n=136)$ (Fig. 1).

Fig. 2, $A$ and $B$ demonstrates that tumor type was not a significant predictor of overall survival ( $p=$ $0.16)$ or disease-specific survival $(p=0.68)$. The median overall survival was 27 months for patients with adenocarcinoma compared with 22 months for those with squamous cell carcinoma. The median disease-specific survival was 29 months for the adenocarcinoma group compared with 31 months for the squamous cell carcinoma group. Furthermore, overall and disease-specific survivals were not significantly different according to cell type when adeno- 


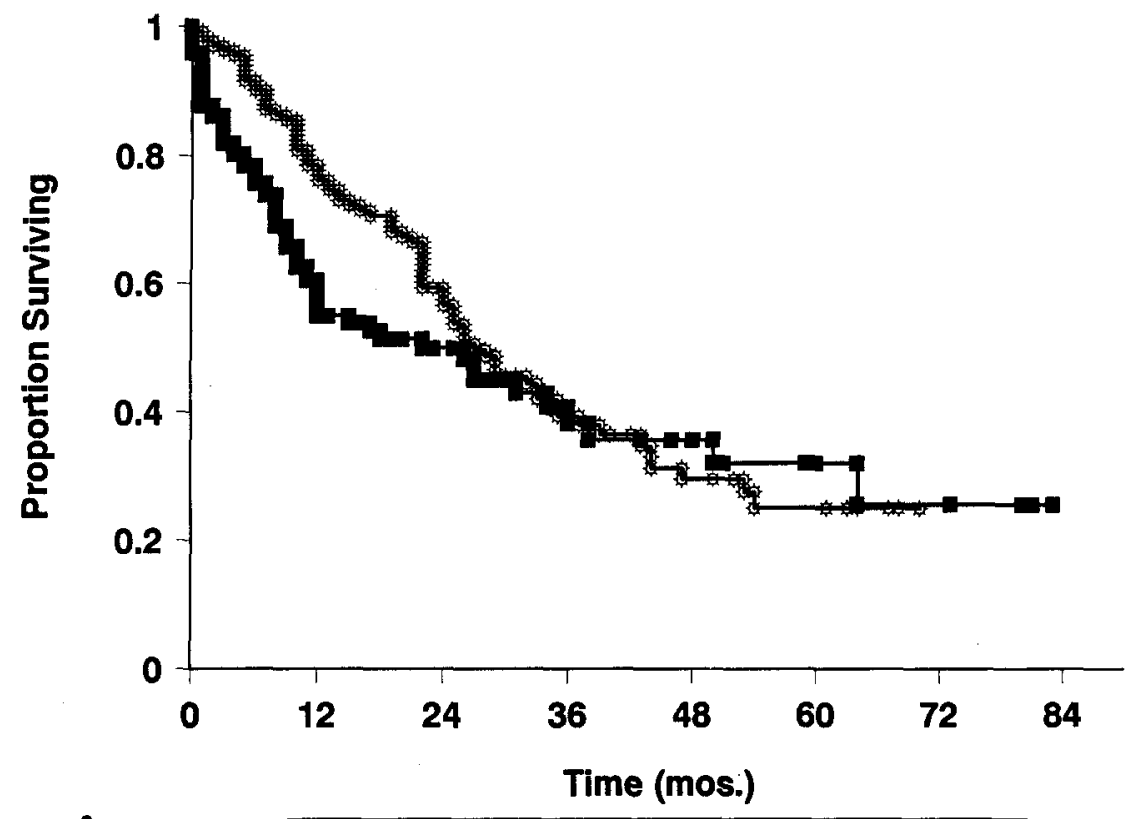

A $\quad$ ADENO $(n=134)$-SQUAMOUS $(n=124)$

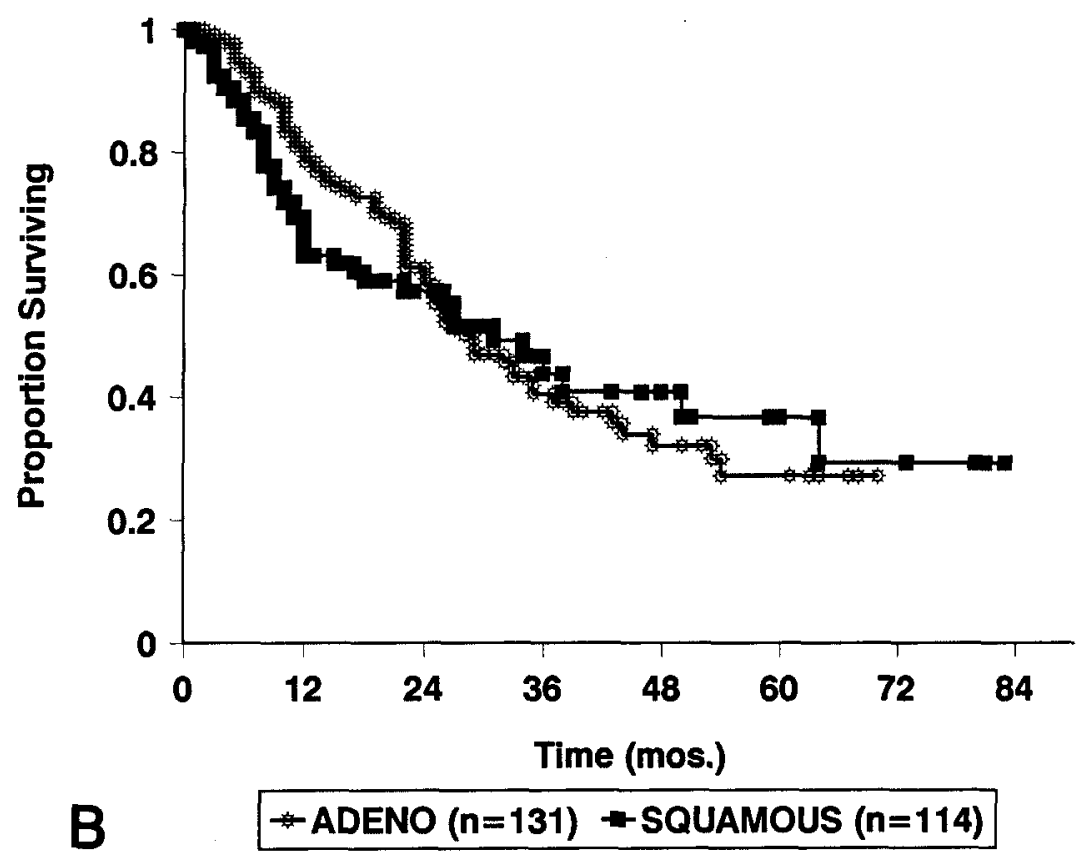

Fig. 2. A, Overall survival according to tumor type, $p=0.16$. B, Disease-specific survival according to tumor type, $p=0.68$.

carcinoma was subdivided into adenocarcinoma arising in the presence $(n=36)$ or absence $(n=98)$ of Barrett's esophagus. The median overall survivals for patients with Barrett's adenocarcinoma, nonBarrett's adenocarcinoma, and squamous cell carci- noma were 28, 27, and 22 months, respectively (Fig. 3).

Table II illustrates the significant prognostic variables identified by univariate analysis. $\mathrm{T}$ stage, $\mathrm{N}$ stage, number of diseased nodes, tumor differenti- 


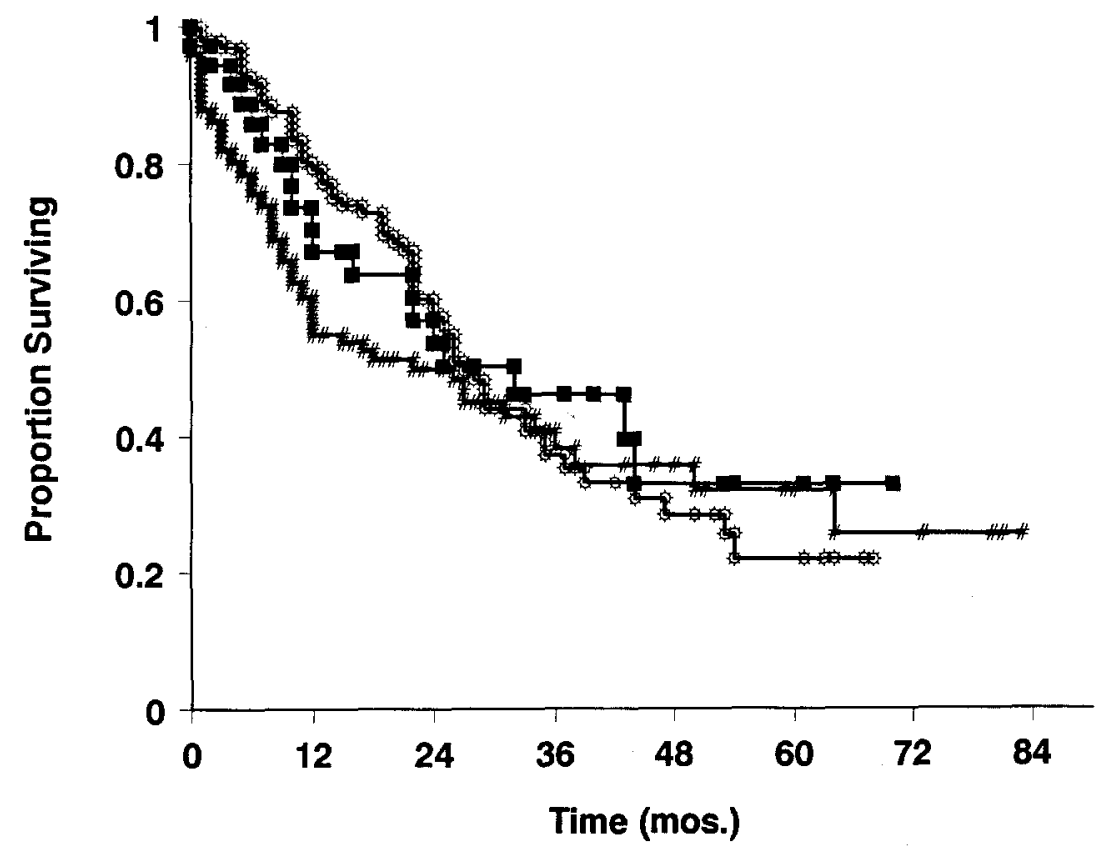

\#Adenocarcinoma $(n=98)$-Barrett's $(n=36)$ \#Squamous cell $(n=124)$

Fig. 3. Overall survival according to tumor type and Barrett's esophagus (Barrett's versus non-Barrett's adenocarcinoma, $p=0.80$; Barrett's versus squamous cell, $p=0.41$; and non-Barrett's adenocarcinoma versus squamous cell, $p=0.26$ ).

ation, tumor site, and blood transfusion were significant variables in predicting overall survival. Of note, tumor type, age, sex, dysphagia, tobacco use, alcohol use, weight loss, Barrett's esophagus, operative procedure, margins, splenectomy, and anastomotic leak were not significant prognostic factors. Preoperative chemotherapy or radiation therapy did not improve survival in patients undergoing esophagogastrectomy for squamous cell carcinoma. Patients with adenocarcinoma did not receive preoperative adjuvant therapy. The median overall survivals for no preoperative therapy, preoperative chemotherapy, and preoperative radiation therapy were 34,12 , and 11 months, respectively.

All demographic information, tumor characteristics, and treatment-related variables were then examined in a Cox proportional hazards model (Table III). Multivariate analysis confirmed the importance of stage and number of diseased lymph nodes on overall survival. The 3-year overall survivals for T1 $(n=22), \mathrm{T} 2(n=50)$, and T3 $(n=186)$ tumors were $64 \%, 56 \%$, and $30 \%$, respectively $(p<0.006)$ (Fig. 4). Patients without lymph node metastases $(n=103)$ survived longer than patients with lymph node metastasis $(n=155)$ (3-year survival; normal
Table III. Multivariate analysis of overall survival after esophagogastrectomy for carcinoma of the esophagus or gastroesophageal junction

\begin{tabular}{lc}
\multicolumn{1}{c}{ Variable } & $p$ Value (Cox) \\
\hline T stage & 0.006 \\
N stage & 0.01 \\
No. of diseased nodes & 0.03 \\
Blood transfusion & 0.07 \\
Splenectomy & 0.16 \\
Histologic cell type & 0.17 \\
Tumor site & 0.27 \\
Preop. chemotherapy & 0.48 \\
Weight loss & 0.52 \\
Tumor differentiation & 0.66 \\
Age & 0.70 \\
Anastomotic leak & 0.74 \\
Margin & 0.78 \\
Preop. radiation therapy & 0.81 \\
\hline
\end{tabular}

lymph nodes $=56 \%$, diseased lymph nodes $=27 \%$, $p=0.01$ ) (Fig. 5). Furthermore, increasing number of diseased lymph nodes was an adverse prognostic variable as illustrated in Fig. 6. Three-year overall survivals for patients with normal nodes, one to three diseased nodes, and four or more diseased nodes were $56 \%, 37 \%$, and $18 \%$, respectively $(p=$ 


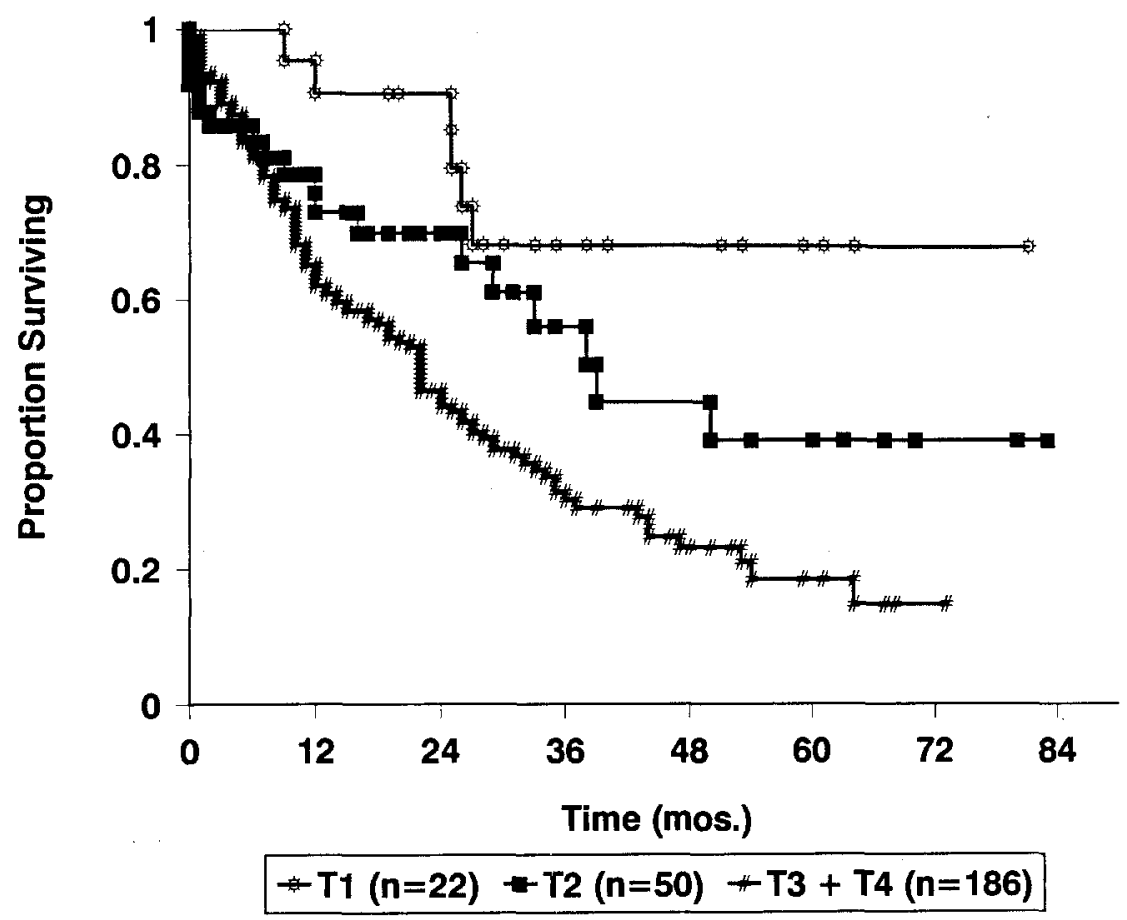

Fig. 4. Overall survival according to $\mathrm{T}$ stage, $p=0.006$.

0.03). The requirement for perioperative blood transfusion approached significance as an adverse factor. Three-year survival for blood transfusion $(n=134)$ was $31 \%$ and that for no transfusion $(n=124)$ was $47 \%(p=0.07)$.

\section{Discussion}

This study demonstrated that outcome after curative esophagogastrectomy is most strongly influenced by extent of disease defined by $\mathrm{T}$ stage, $\mathrm{N}$ stage, and number of diseased lymph nodes. Despite the greater prevalence of adverse prognostic features such as advanced $\mathrm{T}$ stage, metastasis to regional lymph nodes, tumor involvement in more than three lymph nodes, and poorly differentiated tumors, patients with adenocarcinoma and those with squamous cell carcinoma had similar overall and disease-specific survival. Histologic features including cell type, differentiation, and the presence of Barrett's esophagus were not independent prognostic variables.

In contrast to this report, previous investigators reported a survival advantage for patients with squamous cell carcinoma compared with those having adenocarcinoma of the esophagus or gastroesophageal junction. ${ }^{3-10}$ Muller and associates, ${ }^{3}$ in a review of 1201 papers on surgical treatment of esophageal cancer from 1980 to 1988, reported a lower 5-year survival for patients with adenocarcinoma than for those with squamous cell carcinoma, $24 \%$ versus $18 \%$. Mathisen and colleagues ${ }^{4}$ reported a 5-year survival of $33.2 \%$ for patients with squamous cell carcinoma $(n=31)$ compared with $7.7 \%$ for those with adenocarcinoma $(n=73)$. A survival advantage for patients with squamous cell carcinoma may have been due to administration of preoperative chemotherapy to patients with squamous cell carcinoma. Lund and colleagues ${ }^{5}$ documented a disparity in 5-year survival between squamous cell $(13 \%)$ and adenocarcinoma $(2 \%)$ of the distal esophagus. Griffith and Davis, ${ }^{6}$ in a study of distal esophageal carcinoma, demonstrated a survival advantage for patients with squamous cell compared with adenocarcinoma. Furthermore, these investigators noted that squamous cell tumors of the middle esophagus had a worse prognosis than distal malignant tumors, 5-year survivals of $25 \%$ versus $16 \%$. Matthews and Steel, ${ }^{7}$ in a small series of patients with resected carcinoma of the esophagus or gastroesophageal junction, reported a 5-year survival of $28 \%$ for squamous cell $(n=39)$ and $12 \%$ for adenocarcinoma $(n=33)$. The authors also noted that regardless of histologic cell type, patients with lymph node metastases had a poor survival. A large 


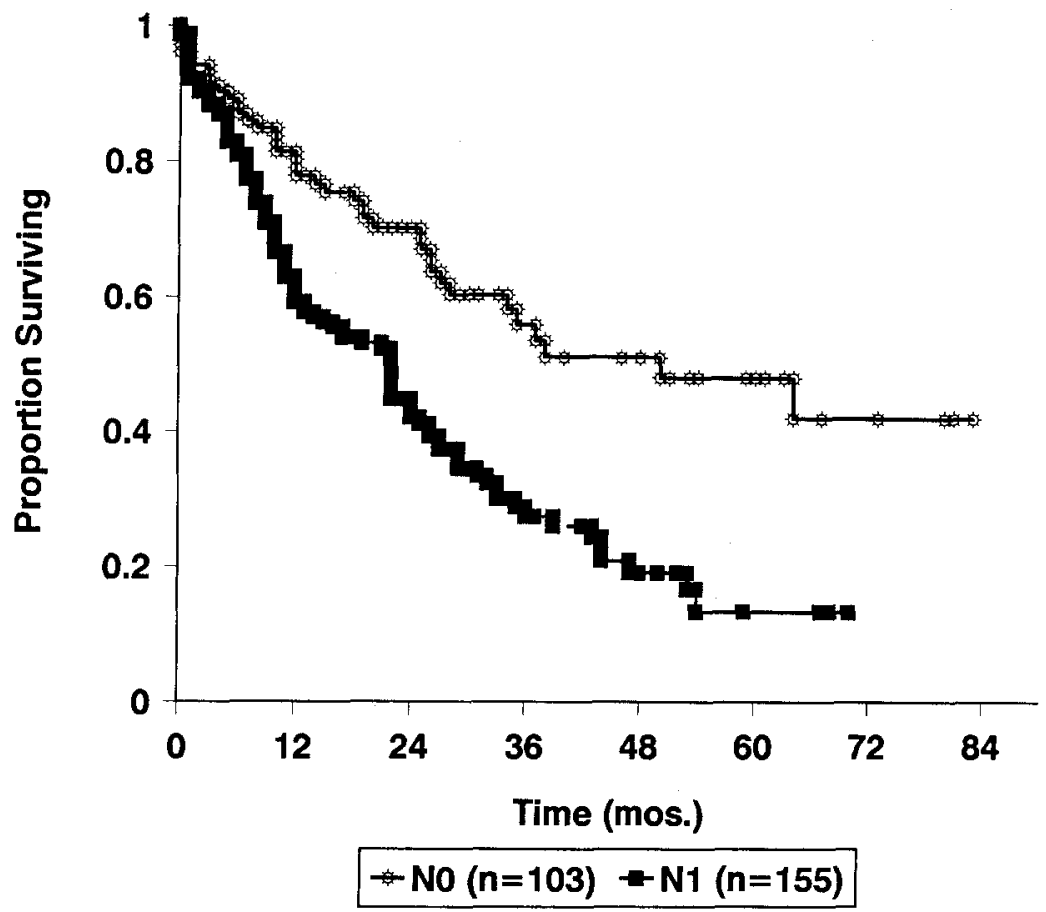

Fig. 5. Overall survival according to $\mathrm{N}$ stage, $p=0.01$.

series from China compared overall survival for patients undergoing resection for tumors of the esophagus $(n=2032)$ and cardia $(n=1649) .{ }^{8}$ The 5-year survival was $47 \%$ for tumors of the esophagus and $24 \%$ for tumors involving the cardia. Unfortunately, information on histologic cell type was not available, but a reasonable assumption would be that most tumors of the esophagus were squamous cell and tumors of the cardia were adenocarcinoma.

Only one study, a multicenter retrospective analysis of 2400 patients with tumors of the esophagus and cardia, reported a survival advantage for patients with adenocarcinoma compared with squamous cell carcinoma, 5-year overall survivals of $27 \%$ and $16 \%$, respectively. ${ }^{15}$ The studies demonstrating the prognostic significance of histologic cell type were univariate analyses summarizing in some instances three decades of an institution's experience with carcinoma of the esophagus and including patients with noncurative resections. Thus one must be cautious in interpreting the significance of histologic type on the basis of these data.

Several studies indicated histologic cell type was not of prognostic significance. Skinner, Dowlatshahi, and DeMeester, ${ }^{16}$ in an analysis of "favorable" (T1, T2, or N0) carcinomas, reported that wall penetration and lymph node involvement were independent factors, but cell type and differentiation were not prognostic. This report, which is in concordance with our data, noted a more advanced stage at the time of diagnosis for patients with adenocarcinoma compared with squamous cell carcinoma. Another univariate analysis comparing adenocarcinoma of the gastroesophageal junction $(n=108)$ with squamous cell carcinoma $(n=104)$ of the esophagus had equivalent 5-year survivals, $18 \%$ versus $19 \%$, respectively. ${ }^{17}$ Three multivariate analyses controlling for stage failed to demonstrate prognostic significance of histologic cell type. Ellis, Gibb, and Watkins ${ }^{18}$ confirmed the significance of stage, but cell type, age, sex, site, and duration of symptoms were not independent factors. A multivariate analysis of 100 patients with esophageal cancer demonstrated that stage and blood transfusion were independent prognostic factors, but cell type was not significant. ${ }^{19}$ Lerut and associates, ${ }^{20}$ in an analysis of 257 patients with esophageal cancer, reported that TNM stage and lymph node status predicted survival. No significant differences were observed according to tumor location, pathologic type, sex, or age. ${ }^{20}$ Thus all studies in which multivariate analyses of prognostic factors were used demonstrated that histologic cell type, including Barrett's esophagus, is not an independent predictor of survival for patients with carcinoma of the esophagus or gastroesophageal junction. 


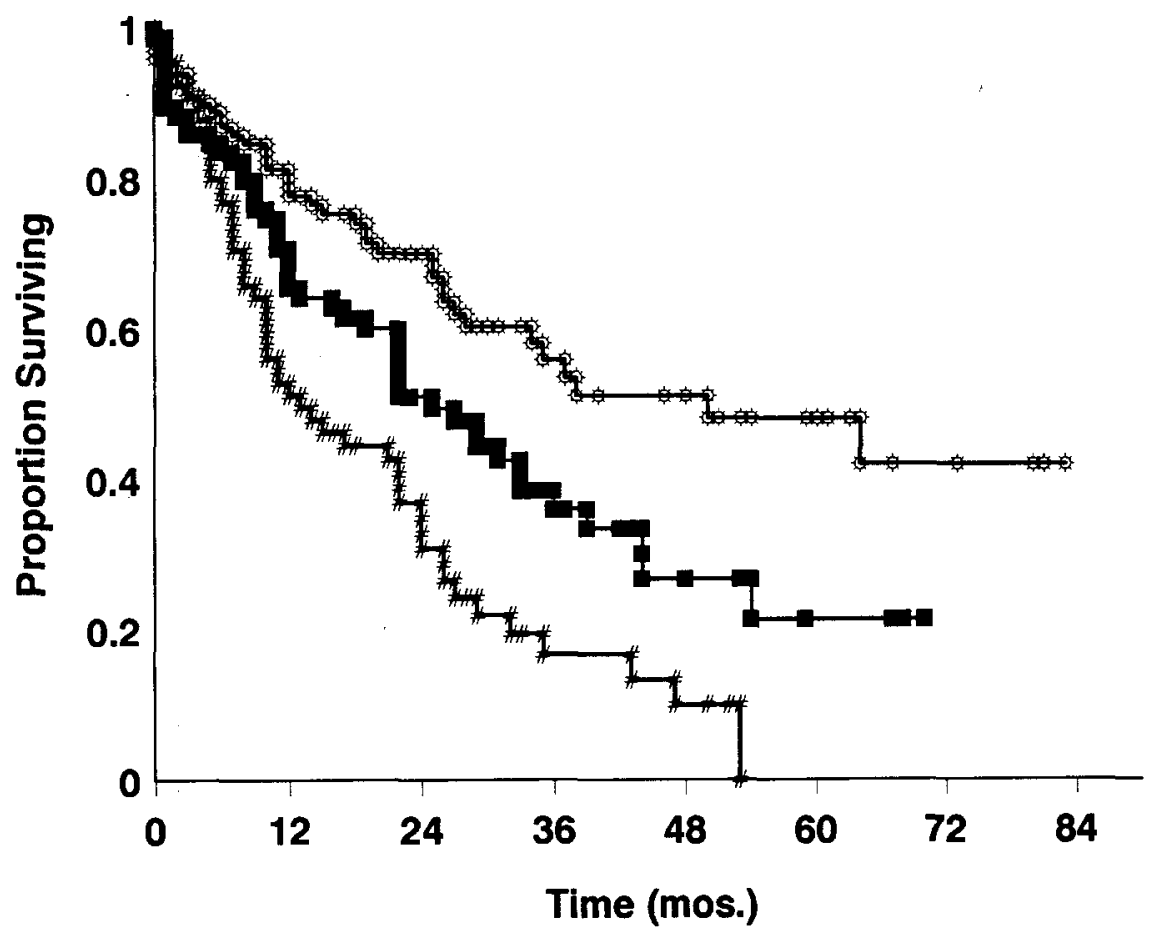

\# Node neg. $(n=103)$-Nodes $1-3(n=87)$ \#odes $\geq 4(n=68)$

Fig. 6. Overall survival according to number of diseased lymph nodes, $p=0.03$.

Although histologic cell type was not a significant prognostic variable, poor prognostic factors were prevalent in patients with adenocarcinoma: advanced $T$ stage, lymph node metastases, and more than three diseased lymph nodes. One possible explanation is that the lymph node dissection for gastroesophageal lesions, which are predominately adenocarcinomas, may have been more complete than for intrathoracic squamous cell lesions. Thus some of the N0 squamous cell carcinomas may actually have been N1. Another explanation is that some patients $(n=33), 29 \%$, with squamous cell carcinoma, in contrast to none of the patients with adenocarcinoma, received preoperative chemotherapy or radiation therapy, or both. This may result in preoperative downstaging of squamous cell carcinoma. Endoscopic ultrasonic (EUS) evaluation was performed in 10 of the 33 patients receiving neoadjuvant therapy. In our experience EUS has a greater than $90 \%$ concordance rate for $\mathrm{T}$ stage for tumors of the esophagus. ${ }^{21}$ On initial EUS evaluation before neoadjuvant therapy, all 10 patients had EUS T3 lesions. After neoadjuvant therapy and resection, six of 10 patients had pathologic T3 tumors. If one accepts that EUS is accurate for the assessment of T stage for esophageal cancer, then in $40 \%$ of patients in whom EUS was performed the tumor was downstaged after preoperative chemotherapy or radiation therapy, or both. Thus downstaging as a result of neoadjuvant therapy may account for the disparity in pathologic stage between squamous cell carcinoma and adenocarcinoma.

Although neoadjuvant therapy may have downstaged some squamous cell carcinomas, our results did not show a survival advantage for patients treated with chemotherapy or radiation therapy, or both. Because preoperative chemotherapy may improve survival, ${ }^{22}$ an additional analysis of patients receiving chemotherapy was performed. All patients receiving preoperative chemotherapy had T3 or N1 disease clinically. Thus a comparison between patients with squamous cell carcinoma receiving preoperative chemotherapy $(n=25)$ versus no preoperative chemotherapy and a T3 lesion $(n=65)$ or no preoperative chemotherapy and $\mathrm{N} 1$ disease $(n=50)$ would be appropriate to estimate the impact of preoperative chemotherapy. The median overall survival for patients with squamous cell carcinoma 
having preoperative chemotherapy was 12 months; for those having no preoperative therapy and a T3 lesion, 17 months; and for those having no preoperative therapy, 12 months. No significant difference in overall survival was noted among these treatment groups.

Adenocarcinoma of the gastroesophageal junction is becoming more prevalent in our center, as well as in other institutions. However, histologic cell type, including the presence of Barrett's esophagus, is not an independent prognostic factor. Patients with tumors of the esophagus or gastroesophageal junction typically have a poor outcome, but a highly selected group of patients undergoing curative resection may enjoy long-term survival. In this series, 3-year actuarial overall survival was $80 \%$ for stage 1 , $48 \%$ for stage $2 \mathrm{~A}, 26 \%$ for stage $2 \mathrm{~B}$, and $29 \%$ for stage 3 disease. We have clearly demonstrated the importance of $\mathrm{T}$ and $\mathrm{N}$ staging in this disease. Furthermore, the number of diseased lymph nodes identifies subsets of patients with more aggressive disease and requirement for more aggressive therapy. The impact of preoperative chemotherapy is unclear, but novel therapeutic strategies and enrollment of patients in prospective trials must continue, particularly for patients with $\mathrm{T} 3$ or N1 disease.

\section{REFERENCES}

1. Blot WJ, Devesa SS, Kneller RW, Fraumeni JF. Rising incidence of adenocarcinoma of the esophagus and gastric cardia. JAMA 1991;265:1287-98.

2. Zheng T, Mayne ST, Holford TR, et al. The time trend age-period-cohort effects on incidence of adenocarcinoma of the stomach in Connecticut from 1955-1989. Cancer 1993;72:330-40.

3. Muller JM, Erasmi H, Stelzner M, Zieren U, Pichlmaier $H$. Surgical therapy of oesophageal carcinoma. Br J Surg 1990;77:845-57.

4. Mathisen DJ, Grillo HC, Wilkins EW, Moncure AC, Hilgenberg AD. Transthoracic esophagectomy: a safe approach to carcinoma of the esophagus. Ann Thorac Surg 1988;45:137-43.

5. Lund O, Hasenkam JM, Aagaard MT, Kimose HH. Time-related changes in characteristics of prognostic significance in carcinomas of the oesophagus and cardia. Br J Surg 1989;76:1301-7.

6. Griffith JL, Davis JT. A twenty-year experience with surgical management of carcinoma of the esophagus and gastric cardia. J THORAC CARdiovasC SURG 1980; 79:447-52.

7. Matthews HR, Steel A. Left-sided subtotal oesophagectomy for carcinoma. Br J Surg 1987;74:1115-7.
8. Shao L, Gao Z, Yang N, Wei G, Wang Y, Cheng C. Results of surgical treatment in 6,123 cases of carcinoma of the esophagus and gastric cardia. J Surg Oncol 1989;42:170-4.

9. Sugimachi K, Inokuchi K, Kuwano H, Kai H, Okamura T, Okudaira Y. Patterns of recurrence after curative resection for carcinoma of the thoracic part of the esophagus. Surg Gynecol Obstet 1983;157:53740.

10. Earlam R, Cunha-Melo JR. Oesophageal squamous cell carcinoma. 1. A critical review of surgery. $\mathrm{Br} \mathrm{J}$ Surg 1980;67:381-90.

11. Beahrs OH, Henson DE, Hutter RVP, Kennedy BJ. Digestive system: esophagus. In: Beahrs OH, Henson DE, Hutter RVP, Kennedy BJ, eds. Manual for staging of cancer. 4th ed. Philadelphia: JB Lippincott, 1992:57-9.

12. Kaplan EL, Meier P. Nonparametric estimation from incomplete observations. Am Stat Assoc J 1958;53: 457-81.

13. Peto R, Pike MC. Conservatism of the approximation $\Sigma(\mathrm{O}-\mathrm{E})^{2} / \mathrm{E}$ in the logrank test for survival data or incidence data. Biometrics 1973;29:579-84.

14. Cox DR. Regression models and life tables. J R Stat Assoc 1972;29:187-220.

15. Giluli R, Gignoux M. Treatment of carcinoma of the esophagus: retrospective study of 2,400 patients. Ann Surg 1980;192:44-52.

16. Skinner DB, Dowlatshahi KD, DeMeester TR. Potentially curable cancer of the esophagus. Cancer 1982; 50:2571-5.

17. Hennessy TPJ, Keeling P. Adenocarcinoma of the esophagus and cardia. J THORAC CARdiovasc SuRG 1987;94:64-8.

18. Ellis FH, Gibb PS, Watkins E. Esophagogastrectomy: a safe, widely applicable, and expeditious form of palliation for patients with carcinoma of the esophagus and cardia. Ann Surg 1983;198:531-40.

19. Gertsch P, Vauthey JN, Lustenberger AA, Friedlander-Klar H. Long-term results of transhiatal esophagectomy for esophageal carcinoma. Cancer 1993;72: 2312-9.

20. Lerut T, De Leyn P, Coosemans W, Van Raemdonck D, Scheys I, LeSaffre E. Surgical strategies in esophageal carcinoma with emphasis on radical lymphadenectomy. Ann Surg 1992;216:583-90.

21. Botet JF, Lightdale C, Zauber AG, et al. Endoscopic ultrasonography in the preoperative staging of esophageal cancer: a comparative study with dynamic CT. Radiology 1991;181:419-26.

22. Roth JA, Pass HI, Flanagan MM, Graeber GM, Rosenberg JC, Steinberg S. Randomized clinical trial of preoperative and postoperative adjuvant chemotherapy with cisplatin, vindesine, and bleomycin for carcinoma of the esophagus. J THORAC CARDIOVASC SURG 1988;96:242-8. 


\section{Discussion}

Dr. Zwi Steiger (Detroit, Mich.). In search of common identifiable factors that may contribute to long-term survival, we reviewed the charts of patients with squamous cell carcinoma of the esophagus between the years 1977 and 1985. During this period 7.3\% (37/507) patients survived 5 years or longer. A total of 130 patients had no treatment because of advanced disease. Among those treated, $27.6 \%(104 / 377)$ had a combination of chemotherapy, radiation therapy, and surgery; $6.1 \%$ (23/377) had surgery only; $18 \%$ (68/377) had radiation therapy only; and $48.3 \%$ (182/377) had chemotherapy and radiation therapy. Twenty-four percent (25/104) from the first group, $13 \%$ (3/23) from the second, $2.9 \%(2 / 68)$ from the third group, and $3.8 \%(7 / 182)$ from the fourth group were long-term survivors. I am aware that it is not possible to draw conclusions from this nonrandomized study except that in the first group a complete response to the combination of chemotherapy and radiation therapy conferred a better chance for long-term survival.
Dr. Jeffrey H. Peters. (Los Angeles, Calif.). I want to comment about the prognostic significance of the number of diseased nodes. As you know, the present staging system does not take into account the number of involved nodes, with N0 disease being no involved nodes and N1 disease being any involved nodes present. We have used an alternate staging system for this reason (WNM), believing that an intermediate group with between zero and four nodes is an important group of patients and can be differentiated prognostically. Your data also demonstrates this fact. Perhaps this present staging system should be modified.

Dr. Robert J. Ginsberg (New York, N.Y.). In response to Dr. Peters, if one reads the instructions in the TNM staging system manuals, one will see that anyone can add an " $A$ " and a " $\mathrm{B}$ " to any stage if there is a prognostic factor that is not listed there. Certainly your group, one of the leaders in esophageal surgery, should get in step with the rest of the world, try to conform to TNM staging, and abandon their own staging system.

\section{Availability of JourNaL back issues}

As a service to our subscribers, copies of back issues of THE JOURNAL OF THORACIC AND CARDIOVASCULAR SURGERY for the preceding 5 years are maintained and are available for purchase from the publisher, Mosby-Year Book, Inc., at a cost of $\$ 12.00$ per issue. The following quantity discounts are available: $25 \%$ off on quantities of 12 to 23 , and one third off on quantities of 24 or more. Please write to Mosby-Year Book, Inc., Subscription Services, 11830 Westline Industrial Drive, St. Louis MO 63146-3318, or call (800)453-4351 or (314)453-4351 for information on availability of particular issues. If unavailable from the publisher, photocopies of complete issues are available from University Microfilms International, $300 \mathrm{~N}$. Zeeb Rd., Ann Arbor, MI 48106, (313)761-4700. 